\title{
CELLULAR AUTOMATA AND URBAN STUDIES: A LITERATURE SURVEY
}

\author{
Nuno Norte Pinto \\ npinto@estg.ipleiria.pt \\ Department of Civil Engineering \\ Polytechnic Institute of Leiria \\ Leiria, Portugal
}

\author{
António Pais Antunes \\ antunes@dec.uc.pt \\ Department of Civil Engineering \\ University of Coimbra \\ Coimbra, Portugal
}

Remisión Artículo: 1-2-2007

Palabras Claves: Urban studies, urban modeling, cellular automata.

Resumo: Este artigo apresenta uma pesquisa bibliográfica sobre a técnica matemática de autómatos celulares (CA) e a sua aplicação a estudos urbanos. Os modelos baseados em CA são actualmente alvo de intensa investigação não só em termos teóricos como também na sua aplicação operacional. Diversos modelos são já aplicados a diversas áreas urbanas e regiões metropolitanas em todo o mundo. É feita a apresentação da formulação clássica de CA bem como das suas primeiras aplicações a problemas geográficos e urbanos, sendo ainda discutidas as principais evoluções da técnica. É apresentada uma série de aplicações de modelos baseados em CA e é dedicada uma atenção particular às suas medidas de desempenho e a diversas abordagens de calibração dos modelos.

\section{Introduction}

The use of mathematical tools to model a wide range of spatial problems has been classified for the last decades as an important approach to scientific planning (Batty, 1994). The growth of urban areas is one of the issues which have concentrated a large research effort, and a series of new modeling techniques were introduced and developed throughout the last decades. Before the democratization of the use of computers, back in the 1970s, modeling approaches were usually applied on problems which demanded few information and, consequently, small computational effort. Computers made possible the use of more disaggregate information, shifting models from a large-scale perspective to microsimulation. New techniques were sought and Tobler (1979) proposed the application of a cellular approach for modeling geographic phenomena, introducing the use of cellular automata (from now on referred to as CA) to geography. During the next two decades, a great effort was made to develop CA-based models: Couclelis (1985), White and Engelen (1993), and Batty (2005) worked on their theoretical issues regarding CA application to urban studies, Batty and Xie (1997) and Clarke, Hoppen and Gaydos (1997) worked on the application of important evolutions of CA to real world problems, Semboloni (2000) studied urban infrastructure development, O'Sullivan (2001b) used an integrate approach based on CA and on graph theory to study gentrification, Semboloni (1997) 
and Ward, Murray and Phinn (2003) developed multi-scale urban models based on CA, and Silva and Clarke (2002) and Barredo, Kasanko, McCormick and Lavalle (2003) made applications of previously developed CA models to large metropolitan areas.

The main goal of this paper is to produce an introduction to CA as an urban modelling tool. After introducing the classic formulation of CA in Section 2, the use of CA in urban studies is discussed in Section 3. Section 4 is dedicated to discussing some important evolutions and relaxations of formal CA. In Section 5 a series of CA applications are presented. Section 6 presents a discussion on the most common measures of performance used to evaluate $C A$ models. Finally, in Section 7, different calibration approaches for CA models are discussed.

\section{The concept of cellular automata}

CA were first introduced in the 1940s by John von Neumann, the founder of game theory, and Stanislaw Ulam, who worked in the Manhattan Project and made intensive research in the field of Monte Carlo simulation. Both researchers were dedicated to studying self-reproduction and to modeling biological life, trying to devise a mathematical formulation that could reduce the forces governing reproduction to logical rules (Torrens, 2000). The concept of automatic computation is on the very foundations of CA, as von Neumann was strongly influenced by Alan Turing's Universal Turing Machine, a theoretical concept of a computer capable of produce universal computing $^{1}$ (Wolfram, 2005). This Machine would be able of taking new actions in a given moment of time based both on a set of parameters and on its knowledge about the external world obtained from an input system of any kind².

An automaton can be considered a Turing-like machine that operates over a tessellated cell space. The term automaton refers to a self-operating machine that "processes information, proceeding logically, inexorably performing its next action after applying data received from outside itself in light of instructions programmed within itself' (Levy (1992) cited by Torrens (2000)). However, there is a great difference between CA and Turing's Machine: CA are parallel processors, with a series of rules working at the same time while the Turing Machine is a serial processor that can only handle a process after another (Torrens, 2000).

According to its first formulation for a 2D cellular automata by von Neumann, presented by Benenson and Torrens (2004)), each automaton cell processes information and proceeds in its action considering both a set of transition rules and data received from the environment. Formally, each cell $A$ is defined by a set of cell states $S=\left\{S_{1}, S_{2}, \ldots, S_{N}\right\}$ and a set of transition rules $T$

$$
A \leftarrow(S, T)
$$

Transition rules define an automaton state $S_{t+1}$ at time step $t+1$ depending on its state at time step $t, S_{t}\left(S_{t}, S_{t+1} \in S\right)$ and an input information $I_{t}$ :

\footnotetext{
1 "A system may be regarded as a universal computer if, given a suitable initial program, it is capable of implementing any finite algorithm through its evolution over time, i.e., that it is capable of producing a working copy as complicated as itself, and the means to make further copies" (Torrens, 2000).

${ }^{2}$ In the original formulation the input was an infinitely long tape ruled off into sections where binary information could be read or wrote.
} 


$$
T:\left(S_{t}, I_{t}\right) \rightarrow S_{t+1}
$$

A grid of automata becomes cellular automata when the input is defined by the states of neighboring cells. This definition of neighborhood is basically the set of cells that influences the state of the cell under consideration.

$$
A \leftarrow(S, T, R)
$$

where $R$ denotes automata neighboring $A$ and establishes the boundary for drawing input information I which is necessary for the application of transition rules $T$. Classic neighborhoods such as the von Neumann neighborhood (Figure $1 \mathrm{a}$ ) and the Moore neighborhood (Figure $1 \mathrm{~b}$ ) are commonly used in 2D cellular automata. The first one is a set of four adjacent cells usually located in the main cardinal points. The second one is the entire set of eight adjacent cells. Variations and conjugations of these two types of neighborhood are also referred (as the one depicted in Figure $1 \mathrm{c}$ ).

Figure 1 Classical von Neumann's and Moore's neighborhoods for 2D CA

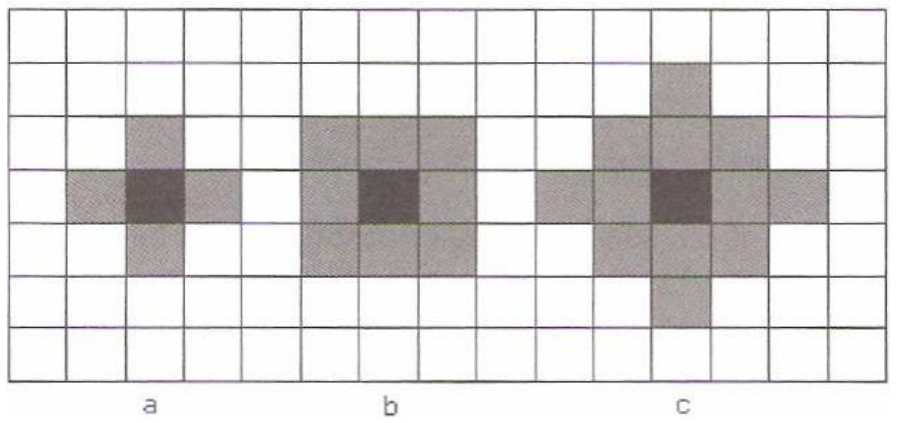

Source: Benenson and Torrens, 2004

The complexity of cellular automata increases as the number of cells increases. Even for few states and small-size neighborhoods, the possible number of transitions is high. Being $N$ the number of cell states and $K$ the number of cells in the neighborhood, the total number of transition rules equals $N^{K}$ (Wolfram, 1983). If the Moore neighborhood (the centre cell and its eight neighbors) is considered for a set of binary states in a 2D CA, the number of different transition rules equals the expressive number of $2^{9}=512$. Von Neumann considered 29 different cell states in his groundbreaking work.

There are two main moments in the history of CA after the introduction of complex systems theory: the discussion over John Conway's Game of Life in 1970 and the work of Stephen Wolfram in the 1980s (Wolfram, 1984). Conway's Game of Life was aimed to design a simple set of rules to study microscopic spatial dynamics of population (Benenson and Torrens, 2004). This 2D CA were based on a set of two cell states, alive (1) or dead (0) and on three transition rules: Survival (Figure $2 \mathrm{a}$ ), Death (Figure $2 \mathrm{~b}$ ) and Birth (Figure $2 \mathrm{c}$ ). The cell remained dead if none of these rules were applied (Figure $2 \mathrm{~d}$ ).

From this simple set of rules, the Game of Life could generate extremely complex patterns of growth through simulation. Furthermore, it could be easily replicated following the concept of the universal Turing Machine. Its main achievement was to formulate a simple interdisciplinary tool 
for representing complex spatial systems and to modeling its dynamics (Benenson and Torrens, 2004). Conway pursued a configuration that could generate moving configurations of stable patterns. After Gardner's challenge on the pages of Scientific American for the research of such a configuration, Robert Gosper and his team at the Massachusetts Institute of Technology implemented a version of Life-CA that generated a machine that could reproduce copies of itself that were as complicated in their structure (Torrens, 2000). Considering the Game of Life as the first widely known CA, and relating its classification as a game to represent the world evolution, it is interesting to consider CA like the world, in its ability of representing the most complex forms of evolution from simple, well-understood interaction rules (Couclelis, 1997). It is worth stating the procedures of Conway's Game of Life as they embodies the key elements of CA (Batty, Couclelis and Eichen, 1997).

Figure 2 Conway's Game of Life set of rules
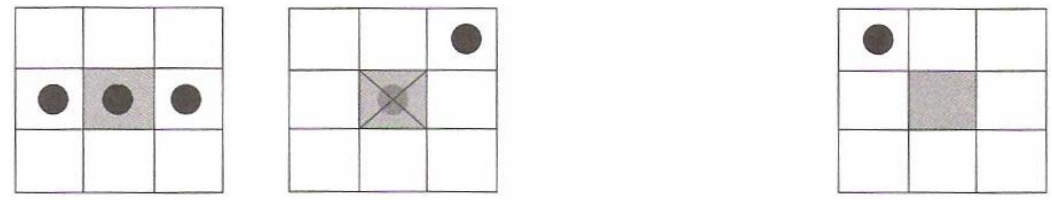

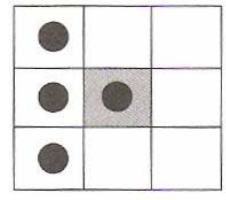

a

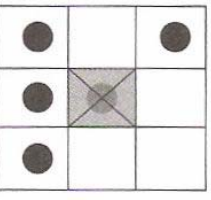

b

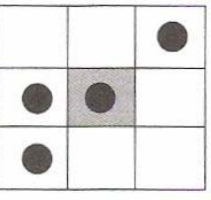

c

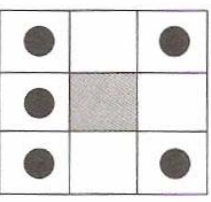

d

Source: Benenson and Torrens, 2004

The introduction of CA as a suitable tool for studying complex systems dynamic made possible through the discussion over Conway's Game of Life, the research on the limits of system's spatial patterns began. The mathematician Stephen Wolfram made extensive research on CA exploring different configurations it could evolve to from simple sets of parameters and rules. Using a simple 1D CA, where the neighborhood is the set formed by the cell and its adjacent neighbors (a set of 3 cells), Wolfram studied limiting patterns for all the possible 256 transition rules $\left(2^{8}\right.$ configurations) (Wolfram, 1983, 1984).

Table 1 Wolfram's classification for 1D CA behavior

\begin{tabular}{clc}
\hline Class & \multicolumn{1}{c}{ CA dynamics evolves towards } & Type of system dynamics \\
\hline I & $\begin{array}{l}\text { Spatially stable pattern - each cell reaches the } \\
\text { stable value of "0" and "1" } \\
\text { Sequence of stable or periodic structures - each } \\
\text { cell changes its states according to the fixed finite } \\
\text { sequence of "0"s and "1"s }\end{array}$ & Limit points \\
II & Limit cycle \\
III & $\begin{array}{l}\text { state is not periodic, but the spatial patterns } \\
\text { repast themselves in time } \\
\text { Complicated localized structures, which are } \\
\text { sometimes long-lived and are more complex than } \\
\text { those of Class III }\end{array}$ & Attractors unspecified \\
\hline
\end{tabular}


Based on numeric experiments, Wolfram demonstrated that limiting configuration of CA does not depend on initial state of cells but it is defined by transition rules. Wolfram classified CA according to their dynamic behavior and the patters generated by them, identifying four main classes described on Table 1 and depicted in Figure 3.

Several other studies tried to devise new classifications by extending the study of Wolfram's classes to larger neighborhoods and different set of rules and parameters. Wolfram's classification has the disadvantage of being impossible to classify a given CA based on the transition rule alone, not knowing what the spatial pattern would be (Benenson and Torrens, 2004). However, Wolfram's classification remains the most popular one.

\section{Figure 3 Typical CA behavior for each of four Wolfram's classes (clockwise from left superior corner: Class I, Class II, Class IV and Class III)}

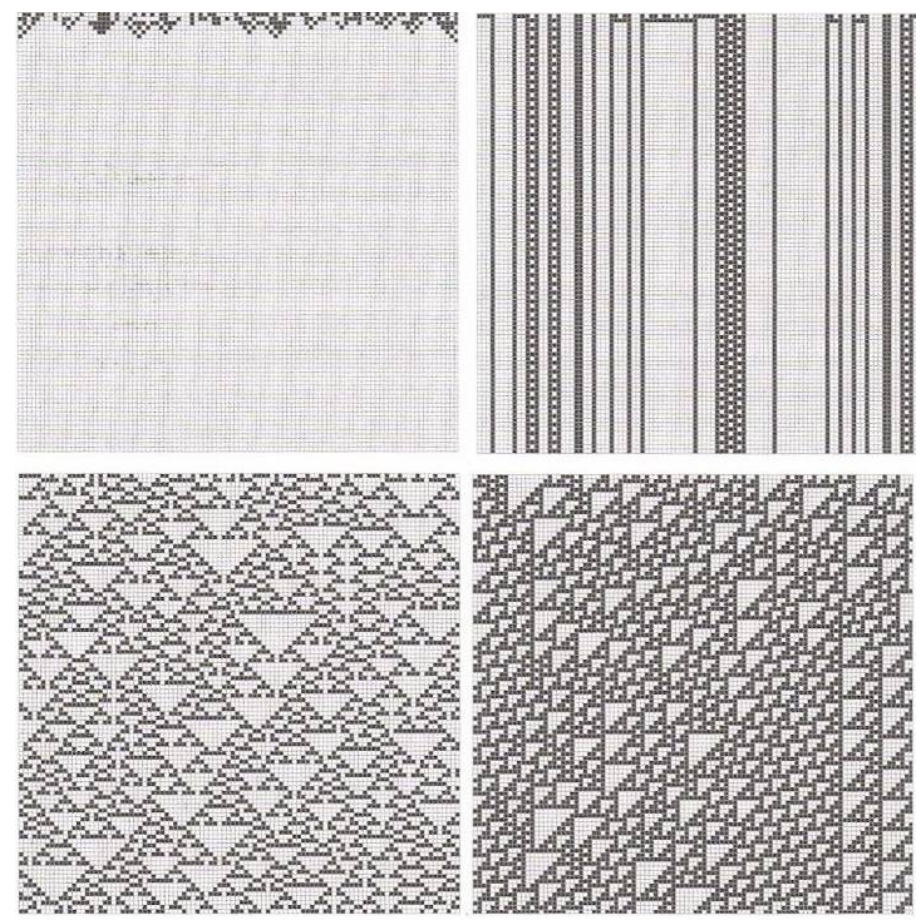

Source: Benenson and Torrens, 2004

It is also important to refer the classification introduced by $\mathrm{C}$. Langton which is very interesting for geography and urban studies (Benenson and Torrens, 2004). He introduces the concept of inactive cells that cannot change its state during CA evolution. It is based on a fraction $\lambda$ of configurations that does not lead the cell to the inactive state (for a more detailed explanation see Benenson and Torrens (2004)). Although a series of new classifications were proposed, several studies on higher-dimensional CA show that their classifications remain similar to Wolfram's classification for 1D CA (Benenson and Torrens, 2004). 


\section{The use of CA in urban studies}

Most geographical theories are static, assuming that the interactions of their agents take place in a market that remains in a state of equilibrium; this assumption is far from being reasonable, as all cities are continually undergoing changes. This fact makes imperative the use of dynamic models, based on the processes that occur in the territory (White and Engelen, 1993). The great attraction of CA is the fact that many classes of system dynamics can be simulated through it; another important feature of CA is its ability to give equal weight to the importance of space, time and system attributes (Batty et al., 1997). The natural ability that CA have to represent complex systems with spatial/temporal behaviors from a small set of simple rules and states made this technique very interesting for geographers and urban researchers. CA are intrinsically spatial and they are used to model a wide range of phenomena due to its ability to represent spatial process, from forest fires to epidemics, from traffic simulation to regional-scale urbanization, polycentricism, gentrification, historical urbanization, urban growth, form and location (Torrens and O'Sullivan, 2001, White and Engelen, 1993). CA-based modeling also allows the integration of socio-economic and natural systems models in a detailed and realistic way (White and Engelen, 1997).

There are three main classes of urban CA models, for three different purposes, not mutually exclusive, that are a direct result of the exploration of modifications of the formal CA: (1) models designed to explore spatial complexity, (2) models designed to research themes of economical, sociological and geographical areas; and (3) models designed to produce operational tools for planning (Torrens, 2000). In this section it will be presented three main studies on the exploration of CA from a theoretical perspective both on its formulation and on spatial complexity. In Section 5 a series of other models will be presented and discussed.

From the 1960s until the mid 1980's geographers were more focused on the study of comprehensive regional models (Benenson and Torrens, 2004). The critics published by Lee (1973) in his "Requiem..." established a transition point where the modeling community started to question the necessity for large scale models and shifted to small scale problems. Problem complexity, even when a small set of spatial relationships was used for modeling purposes, and the lack of experimental data for model calibration generated a search for simpler approaches that could produce more reliable simulation (Klosterman, 1994). CA presented an opportunity to deal with these new modeling requirements. Tobler and Couclelis had produced the grounds for this cellular approach, in the 1970s and 1980s, when it really had a major development along with computer graphics, fractal theory, chaos and complexity (Batty et al., 1997).

Waldo Tobler presented in 1970 his study on population growth simulation based on a cellular model (Tobler, 1970). In this study he invokes the first law of geography ${ }^{3}$ to overcome complexity when dealing with the interdependence of population growth in a given location and its dependence on the population growth everywhere else in the world. Instead of using trend equations with limited set of coefficients that could surrogate the real behavior of growth in every location, he uses an approach that considers the influence on the population growth limited to a neighboring set of locations. Later in 1979 Tobler publishes another important study where CA are explicitly considered to the study of spatial phenomena (Tobler, 1979). Tobler

\footnotetext{
${ }^{3}$ The first law of geography states that everything is related to everything else, but near things are more related than distant things. This is an important concept that supports the link between CA and geography because neighbourhood is one of the main components of CA.
} 
makes a classification of different cell models for land use simulation, taking into consideration its dependence on spatial and temporal issues (Figure 4).

Figure $4 \quad$ Classification of models of land use change

MODEL III

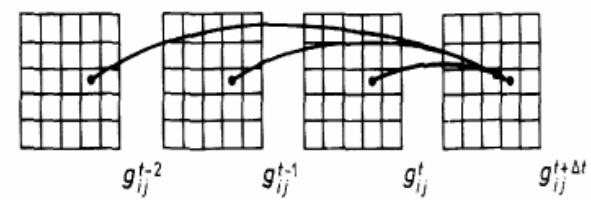

MODEL II

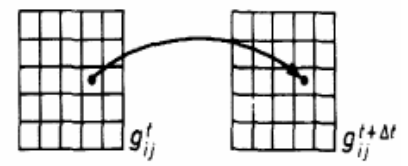

MODEL I
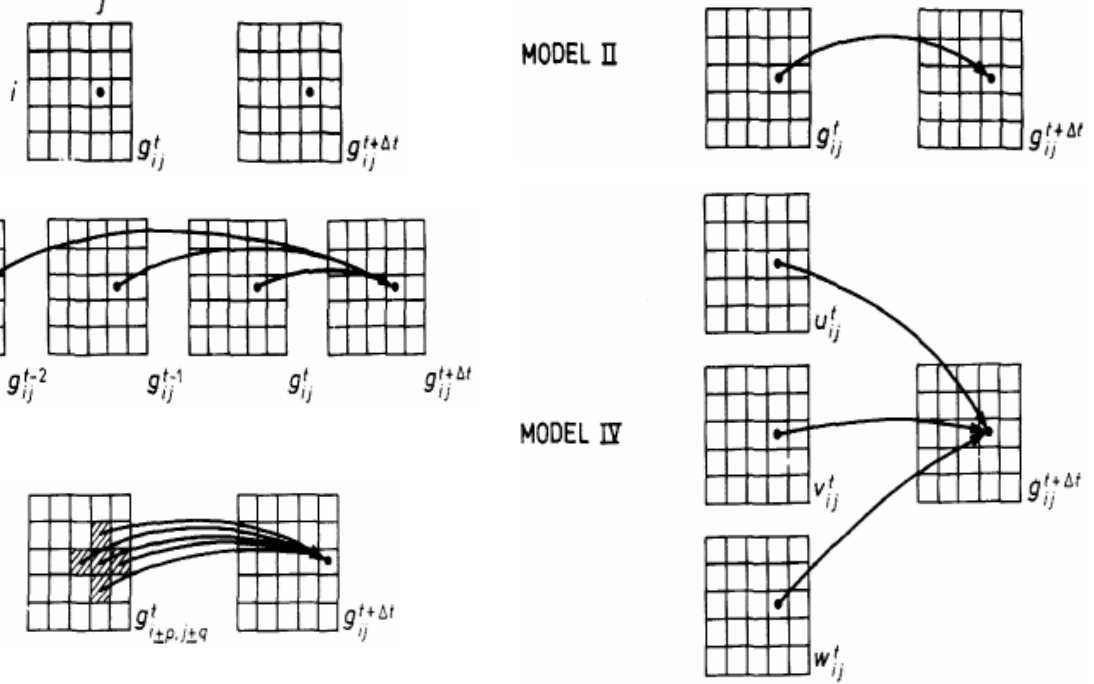

Source: Tobler, 1979

Model type I is an independent model, where the land use for a given location $g_{i, j}$ at time $t+\Delta t$ is not related with the situation at time $t$. Model type II is classified as functionally dependent because land use at a given cell $g_{i, j}$ at time $t+\Delta t$ depends on the land use at that location at time $t$. Model type III is named a historical model as land use of a given location $g_{i, j}$ at time $t+\Delta t$ depends on a series of previous states at previous time steps. Model type IV is classified as multivariate because it depends on several variables other than land use at that location $i, j$. Finally, model type $\mathrm{V}$ is classified as the geographical model because land use at a given location $g_{i, j}$ at time step $t+\Delta t$ is dependent on the land uses of a given neighborhood at time step $t$.

Tobler develops his study using a model type $V$ which he classifies as a dynamic one, because the land use of a given location $g_{i j}$ at time step $t+\Delta t$ is a function $F$ of land use at that location at time $t$ and of a measure of all the land uses in neighborhood $n$ at that same location as expressed in Equation 4:

$g_{i j}{ }^{t+\Delta t}=F\left(g_{i j}{ }^{t}, n_{i j}\right)$.

The neighborhood $n$ is based on the traditional von Neumann neighborhood and is defined as a geographical domain of influence. He considers that, because of different notions of neighborhood different residents have, it should be possible to have dynamic neighborhoods varying on size, shape and orientation.

Transition rules $F$ are defined closely to formal CA definition. Tobler is more interested in studying geographical features of transition rules such as spatial isotropy and spatial stationarity. By spatial isotropy it is meant that the positioning of neighbors does not have any influence in the transition rule. By spatial stationarity it is meant that the same environment, the same neighborhood, results in the same consequences, this is, the rules do not depend on 
where you are. These conclusions are, of course, very generic and considerably distant from real world geographic behaviors (Tobler, 1979).

Tobler's groundbreaking work was followed by a series of other studies that explored CA and its application to geography (Couclelis, 1985, White and Engelen, 1993). They all refer Conway's Game of Life as a paradigm of the application of CA to complex systems from a set of simple transition rules, but never as an example of a geographic system; they all were somehow far from the formal CA definition of von Neumann (Benenson and Torrens, 2004).

In the mid 1980s Helen Couclelis continues the work started by Tobler in the research of CA for urban modeling purposes (Couclelis, 1985). Her work has two main lines: the first one relates to the conceptual and theoretical linkage with the theory of complex systems; the second one relates with the exploration of possible uses in urban planning (White and Engelen, 1993). She establishes a parallelism between Conway's Game of Life transition rules and urban changing phenomena. A 'live' cell could be interpreted as an urban zone that exceeds some kind of threshold in terms of a given urban function. Transition rule 1 (survival) would then guarantee that this cell would maintain this urban function if two or three neighboring cells also exceeded that threshold. Transition rule 2 (death) would make this cell loose that urban function if four or more neighboring cells also exceeded that threshold because the cell would suffocate or, on the other hand, if there was only one neighboring cell exceeding the threshold (then the cell would die from loneliness). Transition rule 3 (rebirth) would settle this urban function in a cell if there were exactly three neighboring cells that exceeded the threshold. A cell would remain dead if none of the precedent conditions were verified.

However, the inherent simplicity of this formulation makes it inappropriate to simulate real world spatial phenomena. Couclelis identifies a series of shortcomings that limits the ability of cellular models to simulate urban phenomena. Issues regarding space dimension and boundary treatment are pointed out as the first limitations for modeling cellular worlds. The imposition of universal transition rules to an infinite regular cell space collides with an empirical interpretation of the method. A second limitation is related with the regularity of neighborhoods. In order to be representative, neighborhoods should be defined by different shapes and sizes for different cells. Cell regularity and spatial homogeneity within each cell are also issues that make strict CA formulation far from being geographically representative. Finally, it is also important to notice the assumption of space and time invariance for transition rules as well as the closure of the system to external perturbations (other than stochastic behaviors) (Couclelis, 1985). The solution relies on the consideration of relaxations that would enhance the ability of a cell-space approach to simulate real world phenomena without loosing both its identity and its simplicity.

Couclelis reformulates the structure of Conway's Game of Life to obtain a simple and strict geographic CA formulation, a generic structure that can be applied to a series of spatial problems (see Couclelis (1985) for further information). She also states that this formulation can be easily relaxed in order to enhance its simulation capabilities. Couclelis' formulation is independent from cell shape and size and even from the existence of a cell space itself. The cell space concept can be generalized to the point where it describes any discrete time/space model representing components and their interactions (Zeigler (1976) cited by Couclelis (1985)). It is likely that any model of interest to geographers, whether aggregate or disaggregate, continuous or discrete, deterministic or stochastic, quantitative or categorical can be expressed in the same language as the cell-space concept (Couclelis, 1985).

Another important concept was introduced by Roger White and Guy Engelen (White and Engelen, 1993): the concept of constrained CA. Standard CA are modeled with the explicit 
intention of maximizing their generality and as a result they present two main characteristics: (1) they are defined on a homogeneous cell space, overriding the variability of cell characteristics as they exclusively relate cells with their state values, regardless of their location on the cell grid; (2) they are unconstrained, so that the number of cells in each state is determined endogenously by the application of transition rules to the current configuration of cells (White and Engelen, 1997). In their initial work, White and Engelen (1997) make some important assumptions that can be considered an approximation of CA to more likely urban behaviors.

The first assumption is related to the consideration of a small number of hierarchically ordered cell states. There are four cell states, vacant (the lowest state), housing, industrial and commercial (the highest one). A cell in the vacant state can change to any other state but the inverse is not possible (thus the city can only grow, which is unlikely to happen). A cell in the industrial state can only change to commercial state.

Another assumption is related to the use of larger neighborhoods than the common Moore or von Neumann ones. The interaction between cells is then dependent of a larger area of influence, breaking up with the formal concept of local neighborhood and introducing what is commonly named action-at-a-distance. On a strict CA model the changes of state must be local, discarding any action-at-a-distance (Batty et al., 1997). In this case, these relationships are assumed non-monotonous and may present positive values (meaning an attractive relationship) or negative ones (repulsive relationship); theses relationships are depicted in Figure 5.

Finally, transition rules are not based on a simple probability of change but in a composite measure of transition potential. This transition potential is a weighted sum that simulates the behavioral propensities of the actors who determine land use, as expressed in Equation 5 :

$$
P_{i j}=S\left(1+\sum_{h, k, d} m_{k d} I_{h d}\right)
$$

where $P_{i j}$ is the transition potential from state $i$ for state $j, m_{k d}$ is the calibration parameter applied to cells is states $k$ at distance $d, h$ is the index of cells within a given distance zone, $I_{h d}$ is equal to 1 if the state of cell $h$ is $k$ and 0 otherwise, and $S$ is a stochastic perturbation. The stochastic perturbation is calculated through the following expression:

$$
S=1+[-\log (R)]^{\alpha}
$$

where $R$ is a uniform random distribution in the interval ]0,1[ and $\alpha$ is a control parameter for the adjustment of the size of the perturbation. This term has a highly skewed distribution so that most values are near unity and much larger values occur only infrequently. The weighted sum in the transition potential is multiplied by $S$ in order to simulate the stochastic behavior of agents in each component of the transition potential. 


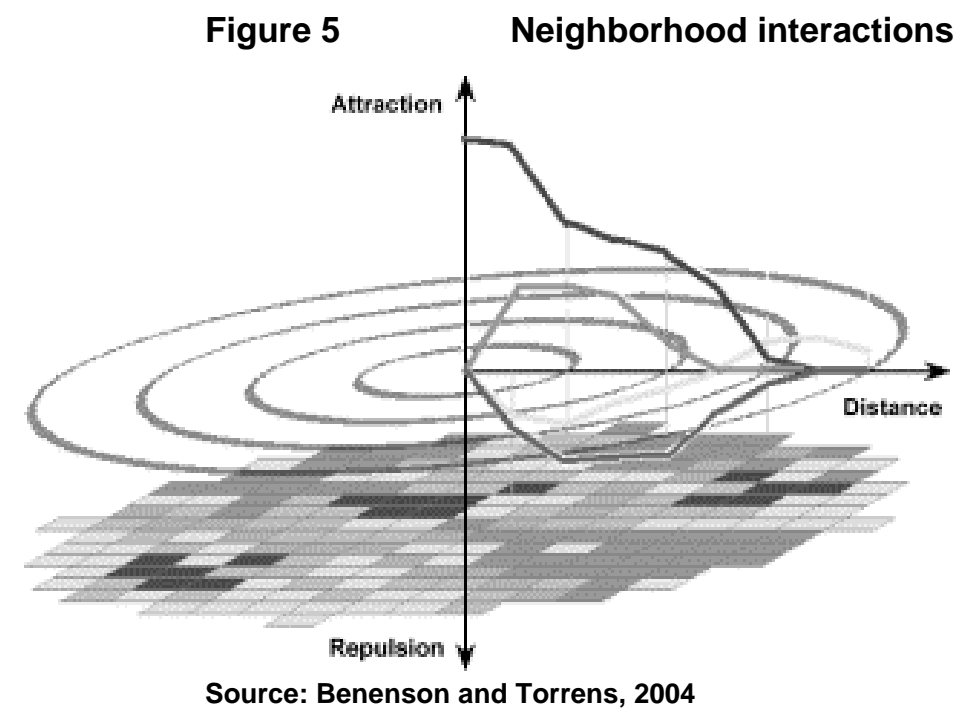

Another important evolution from the early CA models is related with the introduction of land use demand as an exogenous factor. In classic CA, the model evolves over time from a small set of cells to more and more complex systems through transition rules. The number of changeable cells is determined only by the change process itself. White and Engelen (1993) introduced an exogenous parameter that aims to simulate land use demand: there is a predetermined number of cells for each state that is considered in the transition procedure. If the total number of cells is not attained for a given state, there is a probability (which is affected by another calibration parameter) that converts cells in lower cell states to the given cell state until the demand is fully satisfied.

The transition potential is calculated for every cell at every time step of the simulation and is ordered in a descending manner. The set of cells with higher potential is chosen for transition considering land use demand. This approach has been developed and improved and was used in recent studies (Barredo et al., 2003, Pinto, 2006, White and Engelen, 2000). Land use usually depends on three main types of factors: the inherent qualities of land itself, the effects of neighboring land uses and the aggregate level of demand for each land use (White and Engelen, 1997). These components were first explicitly considered by White and Engelen (1993).

Another innovation introduced by this study is the consideration of fractal measures to assess model performance and simulation results. Several fractal measures are calculated for the set of theoretical cities generated by the model and then they are compared with known fractal measures from real world cities such as Cincinnati, Atlanta, and Houston. The fractal dimensions obtained for the theoretical cities are in line with the values observed for the set of real world cities, which indicates CA's ability to produce realistic simulations of urban growth.

It is also important to refer the work of Michael Batty on CA and urban studies. Batty is the author of a series of works where CA is studied both from a theoretical and from an operational point of view. His recently published book Cities and Complexity (Batty, 2005) aims to provide a framework for understanding cities as complex entities and presents CA (as well as agentbased simulation and fractal theories) as powerful tools for simulating this complexity. In his long term work on urban simulation, Batty studied CA as a tool for exploring cities complexity, mostly by formulating and implementing a series of CA models aimed to explore theoretical issues regarding the technique. 
He developed with Yichun Xie models for what they called "areal automata", aimed to simulate urban structure, and for "linear automata" aimed to simulate the growth of a road network with a hierarchy structure (Batty and Xie, 1997). They then generalized these two types of models and developed an urban growth cellular automata model based on cells and networks. These models were based on simple formulations close to the probabilistic formulation of classical CA. The models also considered few land uses which shows their exploratory character. Later a more sophisticated simulator was developed based on the work of Xie. The dynamic urban evolutionary modeling (DUEM) was aimed to capture urban complexity and urban dynamics. It was applied both to theoretical urban structures and to real urban areas, such as Amherst, New York (Xie, 1996). Batty also pioneered the use of CA and GIS as a means of developing powerful simulation tools based on model visualization (Batty, 2005, Batty, Xie and Sun, 1999). The studies presented in this section are considered of great importance to the development of urban CA. They marked the scientific field because they explored CA as technique applied to geographic and urban studies. They also introduced a series of innovations that have successively enhanced the possibilities of using $C A$ as an urban modeling technique.

A series of more recent applications of urban CA are also important to the history of CA and urban modeling. In Section 5 these applications will be presented and their main characteristics and innovations will be discussed. However, it is important to first discuss the main evolutions of CA that are essentially based on relaxations of its four components. The great majority (if not all) of these applications introduced important innovations on urban CA that were aimed to improve its ability of simulating complex urban phenomena. In the next section these relaxations are discussed.

\section{Main relaxations and evolutions}

It was already mentioned that CA have in its simplicity one of its great attractiveness for urban modeling. From a simple set of rules operating over a simple cell structure it is possible to achieve complex forms from simple structures (Torrens, 2000). But the classic formulation of CA is limited in its own formal definition, imposing the necessity of relaxing some of its most basic components. It can be said that the notion "cellular automata" was used in geography, from the beginning, in a very broad sense and not as a rigid formal scheme (Benenson and Torrens, 2004). The formal framework of CA defined by its most known researchers as Ulam, von Neumann, Conway and Wolfram, based on a very simple formulation, is far from being able to represent real cities; many, if not all, urban CA bear little similarity with classical CA. It is questionable whether urban CA still are evolutions of classical CA or are just cellular-based models (Torrens and O'Sullivan, 2001). There are four major adaptations of the strict CA concept: (1) most applications to urban systems relax the neighborhood effect to incorporate action-at-a-distance (considering these as a global consequence of local spatial diffusion, thus reinforcing the application of strict CA); (2) it is hard to identify a scale for urban systems where everything is reducible to one activity in one cell; (3) the need of CA to meet plausible values of change rates; and (4) the use of GIS and map algebra (Batty et al., 1997).

Helen Couclelis pointed out two main characteristics that CA-based models must possess: interactivity and realism (Couclelis, 1997). Interactivity is an essential property that CA-based models must observe. They must allow the evaluation of small changes in the model conditions, to make sensitivity analyses possible (moreover being CA models of complexity, in which a small change in initial conditions or transition rules may produce major changes in the results). This type of models should also incorporate good visualization techniques, not only in the graphic output but also in the statistical characterization of the results. The integration with GIS is pointed out as the next big step, because of the natural affinity between CA and raster 
images, and because of the potential of graphical visualization provided by GIS. However, the more complex is the CA-model structure, the more visualization becomes difficult.

Another important feature of CA-based models is realism. Couclelis argues that no model based on classic assumptions of CA - homogeneity, uniformity, universality - can claim good performance when applied to real world problems (Couclelis, 1997). There are two main dimensions of model realism: realism with respect to data and realism with respect to model structure. The linkage between CA and GIS once again can be very profitable in order to guarantee data realism. Several CA-based models are already supported by GIS features (Batty et al., 1999, Putman and Shih-Liang, 2001, Takeyama and Couclelis, 1997, Wagner, 1997).

Structure realism is achieved through the adaptation of the classic CA structure to the specific needs of simulating complex urban behaviors. CA ability to generate complex patterns from simple cell configurations and through small sets of rules is one of its main attractions, as it was already stated. But in order to generate feasible urban landscapes it is necessary to adapt the formalism of CA to the behaviors that are the subject of this specific area of simulation.

Couclelis (1997) identifies a series of relaxations that can be implemented in order to enhance the ability of a CA-based model to correctly acquire the behaviors of urban areas in general. Figure 6 depicts a series of relaxations that can be implemented for all the four main components of CA.

The majority of CA models presented so far are based on regular square cells forming an orthogonal cell lattice. The main reason is related with land use data availability from remote sensing maps. In order to enhance spatial representativeness it is possible to forget this formality and to use irregular cells as the spatial unit for CA. Tobler refers that there are some analytical advantages in considering the irregular spatial division of political jurisdictions (Tobler, 1979). However, Tobler states that the basic difficulty, of topological type, relies on the fact that these irregular cells do not all have the same number of adjacent cells, thus its neighborhood can not be defined by any simple notational scheme. There are very few studies based on irregular cells (one of the main proposals of the present study). The model developed by Vandergue et al. (2000) is cited by Ménard and Marceau (2005) as the only CA model that uses census tracts as cells. Semboloni (2000) used Voronoi polygons to model urban growth allowing that a spatial partition of a given cell could occur, which invalidates the use of regular cells. O'Sullivan (2001a, O'Sullivan, 2001b) developed another CA-based model integrating CA and graph theory. Benenson and Torrens (2004) refers the existence of CA theoretical studies that used triangular and hexagonal cells (Eloranta, 1997, Gerling, 1990). These attempts showed that no significant improvements were introduced in the performance of CA model.

The cell state set can also be modified to simulate different land uses in different regions of the territory under consideration. This modification has some implications not only in the definition of neighborhoods but also in the establishment of the set of transition rules.

The concept of neighborhood is also susceptible of being modified. There is a problem or representativeness in the first place. The importance of the neighborhood is that it defines the geographical domain of influence (Tobler, 1979). The fact that there are different concepts of neighborhood must be taken into account: a resident of an urban area has a different idea of neighborhood from a resident in a rural area, therefore it is possible to consider the shape, size and form of a neighborhood as a function of the location of its central cell, giving a special attention to neighborhoods in boundary areas (Tobler, 1979). Neighborhoods such as von Neumann's and Moore's are particular indicated to physical phenomena; human interactions 
are more likely to be explained by wider areas of influence (White and Engelen, 2000). Neighborhood structure may also vary in time. There are some studies in the field of tissues and leaves growth that considered the creation of new cells within a given neighborhood (Lindenmayer, 1968). Semboloni (2000) also assumed that cells could be divided, thus creating new neighborhoods.

Ménard and Marceau (2005) produced an interesting study on spatial scale sensitiveness in geographic CA. They gathered information about the neighborhoods used in several CA models from 1993 to 2003. They defined CA spatial scale as a set of three components: spatial extent, cell size and neighborhood configuration. They focused their study on the analysis of different cell sizes and neighborhood configurations for a simple two state problem. Small variations in cell size can produce significant variations in results when a given scale threshold is exceeded. The authors state that CA models are not sensitive to variations of neighborhood configuration. Spatial scale sensitivity affects CA models were the cell actually represents a portion of the geographic space. Ménard and Marceau (2005) also state that although scale issues are supposed to be considered as a design choice that will not drastically vary during the simulation process, modelers must pay more attention to the subject because some of its components are effectively dependent on scale choice.

\section{Figure $6 \quad$ Possible CA relaxations}

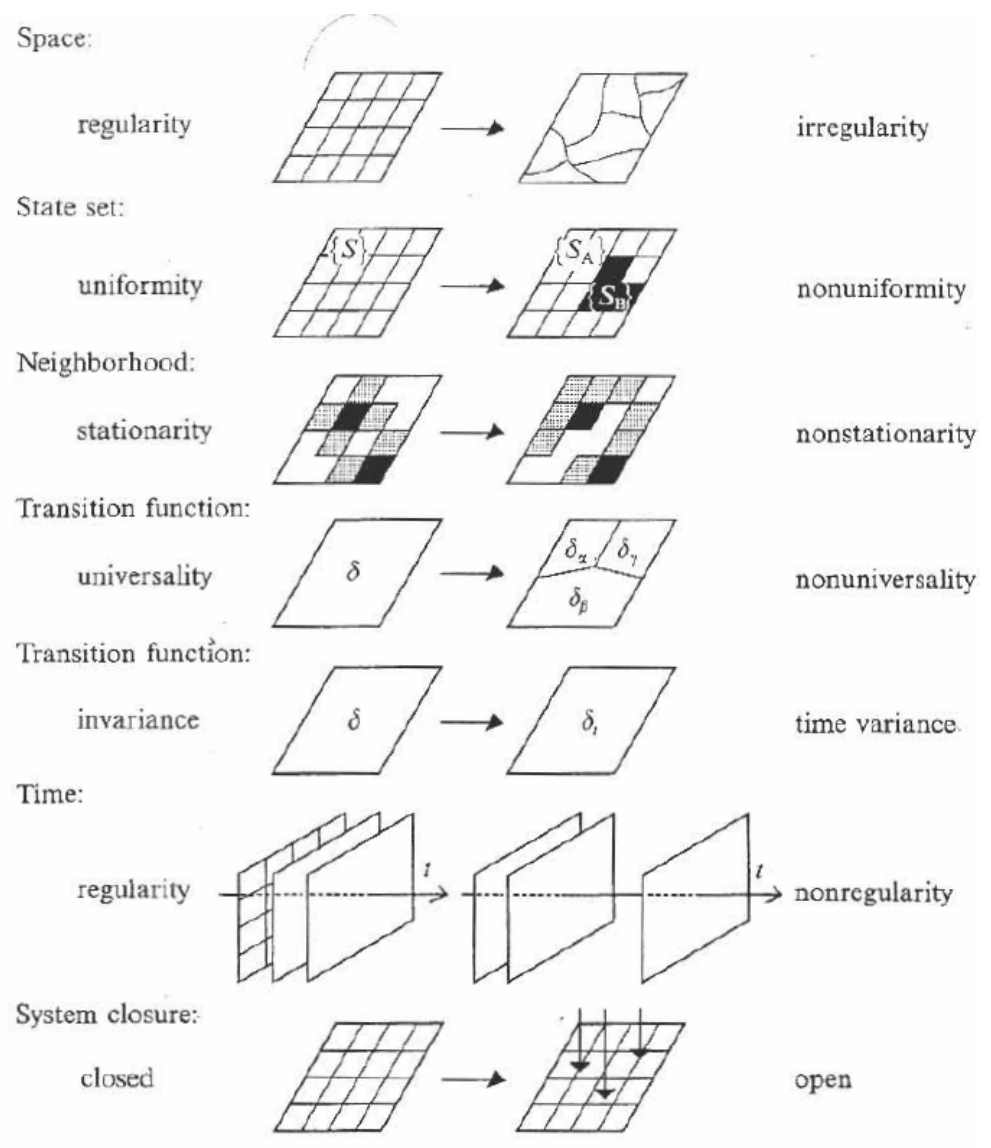

Source: Couclelis,1997 
Transition rules also underwent major developments from their initial classic formulation. The main component of CA is the transition rules (Torrens, 2000). The first and more significant evolution is related with the incorporation of stochastic perturbations in the transition rules. Deterministic behaviors are not suited for the simulation of complex urban phenomena because they can be considered a result of complex interactions between agents. For this reason, many CA models have incorporated stochastic perturbations in their transition rules (Barredo et al., 2003, Clarke et al., 1997, White and Engelen, 1993). Transition rules can also be considered non-static, varying through time. Their universality, an assumption of the classic CA formulation can also be relaxed, as different areas and land uses present different change behaviors. There may be also a distinction regarding the way transition rules operate over time. They can be applied sequentially, updating cell states one after another or in parallel, updating all cells at the same time (Benenson and Torrens, 2004). Von Neumann's self-reproducing CA presented an asynchronous behavior with the cells being updated considering the previous updates generated by the automata. Conway's and Wolfram's approaches were based on synchronous behaviors, with the entire cell set being updated at the same time (Benenson and Torrens, 2004). There are two main processes of asynchronous updating. The cell set can be updated at a moment in time after a predetermined order according to some cell characteristic. Another method is based on a probability of change in a given moment that is a function of the cell's waiting time (Benenson and Torrens, 2004).

Finally, formal CA can be considered as a black box that processes input data towards an output without any interference from the outside world, that is, it can be considered as a closed system. Urban systems are too far from being close; in fact, an urban system is one of the most opened systems. Therefore, the assumption that an urban system modeled by CA can experience exogenous interference - say stakeholders interference or political decisions enhances its ability to simulate urban complexity and, consequently, its representativeness. Many models deal with this relaxation by introducing stochastic perturbations that only occur when certain exogenously defined thresholds are overcome (Clarke et al., 1997).

Relaxations are needed to improve CA's ability to simulate complex urban phenomena. The use of real, disaggregate data made possible the relaxation of any or all the assumptions of classical CA, allowing the models to better fit into the complexity of cities (Couclelis, 1997). However, excessive relaxation of traditional CA assumptions may increase exponentially the difficulties to understand the outcomes of a model, in a comeback to widely criticized largescale simulations (Couclelis, 1997).

\section{Applications of CA in urban change problems}

The use of CA in geography and in urban studies was introduced in Section 3. A group of three groundbreaking studies were presented in order to illustrate the capabilities CA-based models have to simulate complex urban phenomena. The evolution from the classic formulation of CA to modern urban CA-based models was also presented in Section 4, where the main relaxations and evolutions were discussed. In this section a group of representative CA-based models developed during the last decade or currently under development will be presented. However, the list of CA-based models is by far larger than the one presented in this section.

One of the most widespread models is CA-based SLEUTH developed by Keith Clarke to model and predict urban growth (Candau, 2000, Clarke, 2002, Clarke et al., 1997, Silva and Clarke, 2002). This model is aimed to create a high resolution simulation tool for modeling urban growth (Benenson and Torrens, 2004). Its name is an anagram for Slope, Land use, Exxclusion, Urban, Iransportation and $\underline{H}$ ill Shade. 
SLEUTH has two main modules (Clarke, 2002): first an urban growth model; second, an embedded land use model that uses information from urban growth model.

The urban growth model requires five GIS-base inputs that are used in image format: urbanization, land use, transportation, areas excluded from urbanization, slopes, and hill shading for visualization. Urban extents are required for four different time periods for calibration purposes. Urbanization results from an urban seed file and at least two road maps that interact with a slope layer to allow the generation of new urban centers.

It considers a regular grid space, a neighborhood of eight cells and only two cell states: urban and non-urban. It operates five sequential rules of transition: (1) Diffusion, (2) Breed, (3) Spread, (4) Slope resistance, and (5) Road gravity. The growth rate is a sum of five different factors. The diffusion factor determines the overall dispersion of the distribution of single grid cells and of the movement of new settlements outward through the road system. There is a breed factor which is a coefficient which determines how likely a newly generated settlement is to begin its own growth cycle. The spread factor is a coefficient that controls how much outward "organic growth" expansion takes place within the system. Slope resistance is a factor that influences the likelihood of settlement existence on steeper slopes, and Road gravity is a factor of attraction of new settlements onto the existing road system if new areas fall within a given distance of a road (Clarke, 2002). These coefficients generate four different types of subsequent urbanization (Benenson and Torrens, 2004). First, spontaneous growth: any nonurban cell can be urbanized according to a probability inversely proportional to cell slope. Second, generation of new diffusion centers: each spontaneously urbanized cell can become a new spreading centre if it has a given number of neighboring urban cells and reaches a probabilistic threshold defined as a model parameter. Third, diffusion at the edges of urbanized areas: there is a fixed probability (another parameter of the model) that allows an edge cell to become urbanized given a certain number of urban neighboring urban cells. Fourth, roadinfluenced diffusion: a new spreading centre is chosen given its distance to the road network. It can be dislocated along that road in a randomly selected direction for a given distance (another parameter of the model) for a new location where it can "paste" development. Two randomly chosen neighboring cells would then change state.

An important improvement of this model is the consideration of self-modification rules aimed to modify the model's behavior over time in order to allow it to integrate historical conditions by calibration. Intensive growth periods or periods of little or no growth are identified and the model can stochastically overcome these behaviors.

After the first phase of urban growth modeling, SLEUTH will assign land uses to the new urban areas obtained from the first phase of the model. This assignment is made through an embedded model named Deltatron. The main assumption is to consider each cell as a Deltatron, an urban entity that is associated to only one cell state (or land use) (Benenson and Torrens, 2004). These entities evolve during simulation in a way similar to the one described for urban growth, through four stages.

Firstly, cells are selected at random as candidate locations for land use change on the basis of how much urban growth has taken place. Each newly urbanized cell is assumed to induce a potential change in land use and, as a result, determines whether the selected cell will keep the same or change to another land use; this is made through the consideration of a probability that depends on historical change and cell slopes. If a transition occurs and the cell is not a Deltatron already, a new Deltatron is created. Cluster dynamics are defined as an aggregation process of these new Deltatrons and the associated land use transition. The newly transitioned cell acts now as the land use aggregation centre. This process behaves closely to "organic" growth described above. Age is also considered to characterize Deltatrons: as time goes by, Deltatrons get older and are eliminated when their age reaches a given threshold. 
SLEUTH has the credit of introducing the concept of evolving transition rules with the aim of improving the model's ability to retrieve past behaviors. It is also a straightforward model that can be easily calibrated to different urban and regional areas with a small set of calibration parameters. There are already an important number of applications not only in the United States but also in Europe, Africa and South America.

Another innovative approach was proposed by Li and Yeh (2001) They formulated a CA-based model that uses artificial neural networks (ANN) to simulate urban change. It has been used in the Pearl River Delta, China. The set of parameters is obtained automatically by the ANN method, which is made more robust because it uses a back-propagation training procedure.

The authors consider that the traditional CA formulation has serious problems in obtaining consistent parameters values. The method is believed to be suited for dealing with complex interactions between dependent variables without user involvement, a major issue in urban modeling. The model is capable of analyzing relevant data, and to eliminate noise and other redundant data. Another important development of this model is related to transition rules: they are obtained from the ANN training process and not from user definition. The model only needs to be fed with training data. It is also capable of dealing with uncertainty by simulating alternative developments through network training, integrating potential exogenous interventions in the change process whose deduction is not possible from historical data. However some shortcomings need to be overcome. Neural networks proved well for training samples but gave poor results for unseen data sets. Basic model structure is depicted in Figure 7.

David O'Sullivan (2001b) proposed a CA-based model that integrates graph theory to enable research into relationships between spatial structure (represented with graphs) and urban dynamics (simulated by CA), as depicted in Figure 8. The model is based on a partition that need not be space-filling and overlapping spatial elements might be used. In order to study urban phenomena, the entities forming the basis of urban morphology (buildings, blocks, streets, census tracts, or administrative/planning zones) are a natural set of spatial elements to use as the graph vertices. Edges in the graph represent some sort of relationship between vertices, so that any relationships relevant to the model being developed might be used. The neighborhood of each vertex consists of a set of adjacent vertices. Cell states may be defined in a suitable way, considering the land use classes as large as necessary to correctly simulate reality. 


\section{Figure $7 \quad$ CA model based on artificial neural networks}

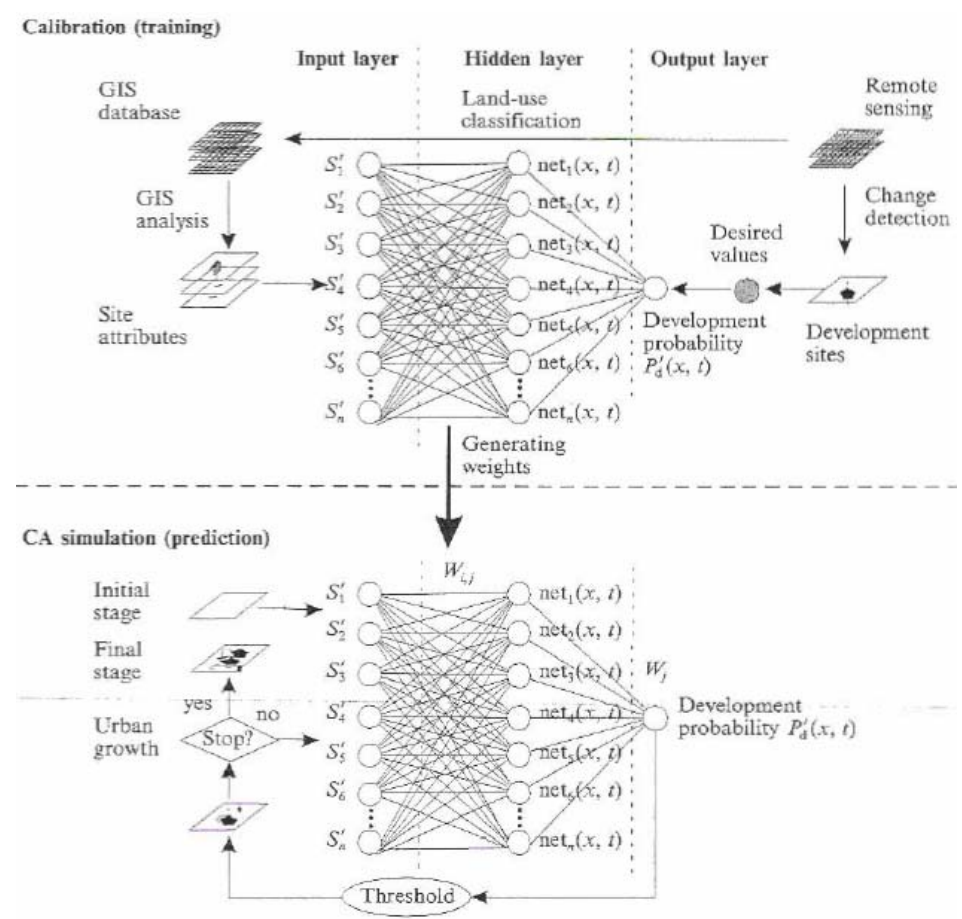

Source: Li and Yeh, 2001

The graph/CA model was applied to study gentrification in small local neighborhoods in central London. Data availability and quality is considered a problem for using this approach to microscale modeling. However, the dynamic behavior of complex models constitutes a major shortcoming to the application of micro-scale modeling to gentrification problems.

Figure 8

Graph-CA model concept

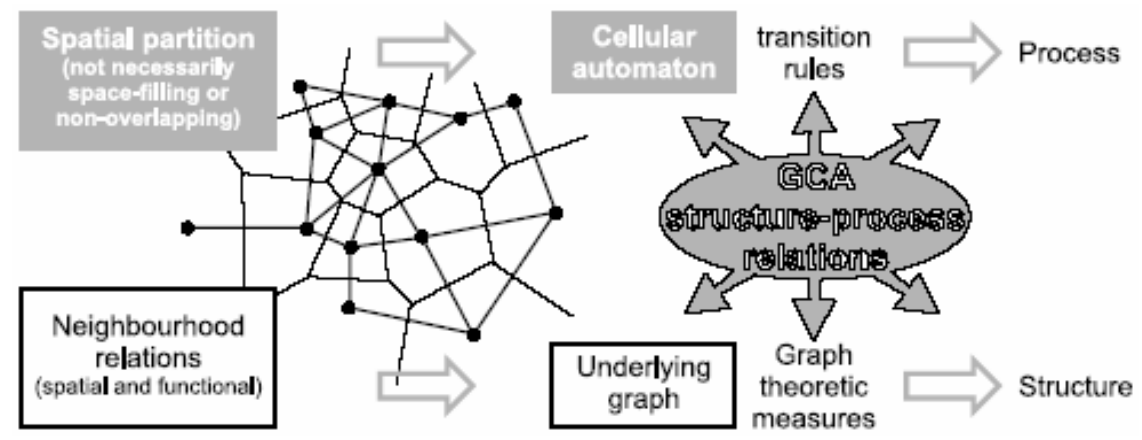

Source: O'Sullivan, 2001b

Following the studies of White and Engelen (1993), Barredo et al. (2003) developed a constrained CA model. They used the concept of transition potential introduced in the former to build a model based on a regular cell grid with a radial neighborhood of 172 cells. They also considered a neighborhood effect materialized through a user-defined weighting factor matrix, establishing quantitative values for attraction and repulsion. 
The model was applied to Dublin considering a thirty-year long historic period (1968 to 1998). Using fractal measures and contingency matrices (discussed in the next section) as goodness of fit functions, the model was able to achieve interesting results. Simulated fractal measures for each land use were quite similar to real values for Dublin and the kValue can be considered satisfactory. However, although the simulation was able to reproduce urban patterns at a macroscopic level, a quick analysis of the graphic output reveals that the model was unable to match land uses for a large number of areas.

Nuno Pinto (Pinto, 2006) proposed a CA model for simulating urban growth of small-size urban areas in the Portuguese context that is also based on the use of irregular cells. The cell space results from the intersection of census tracts (for different reference years) with urban areas, defined by their perimeters. The choice for irregular cells is referred to be more representative of urban structures such as those focused by the study. The model also deals with land use demand through an innovative perspective. Land use demand depends both on the population growth and on the variation of constriction density. These two indicators were considered by using population density (which also varies from one reference year to another) as a measure of land use demand. The model distributes the increase of population over the territory (thus generating the growth of urban areas) rather than sorting out a series of cells for state change based only in a given probability. State transition depends on a measure of transition potential, considered as the calibrated sum of three different components: cell accessibility, land use suitability, and neighborhood interactions between different land uses. The model was applied to a series of theoretical test problems generated to test its behavior. These test problems had the goal of reproducing real-world spatial structures similar to small Portuguese municipalities. The model was also applied to a case study focused on a small municipality. In both cases, the model was able achieve good simulation results when compared with similar studies.

\section{Measurement of CA models performance}

Any modeling activity implies the consideration of a series of measuring procedures to evaluate model performance and to establish the boundaries to its applicability in real world situations.

CA have, as a result of their spatial characteristics, a set of well designed measures that are often applied as performance measures and model fitness functions.

The first and most simple method to evaluate the performance of a CA model is based on the visual comparison between maps from the reference situation at the initial, intermediate and final moments considered on the one side, and maps resulting from the application of the model on the other (Barredo et al., 2003, Clarke et al., 1997, Herold, Couclelis and Clarke, 2005, Ward et al., 2003). Although this method is used as a qualitative procedure to calibrate a CA model to certain conditions as it allows to quickly assess its ability to replicate growth conditions (Clarke et al., 1997), it can not assure a reliable quantitative measure for comparison. Therefore, it can only be used in early phases of the calibration process, with an exploratory purpose. Consequent quantitative measures are needed to correctly evaluate CA-based model performance. Statistical relationships must be derived from a set of model data in conjugation with reference data.

A well known measurement procedure is based on the use of contingency matrices for mapping comparisons (Barredo et al., 2003, Benenson and Torrens, 2004, Couto, 2003). This technique has its origin in image processing science, and is applied in a wide group of different areas of knowledge, from geography to medical imaging.

The basic procedure of this technique is to establish a set of comparison indicators between two maps, one resulting from a modeling process and another serving as a reference for the modeled reality. These two maps can be raster images with a pixel structure or any pair of maps 
that share the same spatial partition, based on regular or irregular units (or cells). The comparison is made through a measure of agreement between the pair of maps. Both maps must be classified by the same finite set of classes, each class associated to a unique land use. The number of cells in each class is then accounted for both maps, and the comparison matrix is assembled. A series of measures is then calculated in order to estimate an indicator of agreement named kappa value.

The contingency matrix is computed confronting the model outcome with the corresponding reference layout, accounting the total number of cells $n_{i j}$ that were modeled in a particular state $i$ when they are referenced as being in state $j$. These two classifications, modeled situation and reference one are independent one from another. The matrix is usually assembled considering the modeled map represented in rows and the reference map represented in columns, as showed in Table 2.

The contingency matrix is an effective way to assess map accuracy, not only globally but also considering each cell state (Couto, 2003). The evaluation of the overall accuracy represented as the sum of the main diagonal of the matrix (Equation 9) is complemented with the evaluation of the producer's accuracy (Equation 10) and the user's accuracy (Equation 11). However, these measures must always be presented along with the contingency matrix, so their values can be compared with the distribution of cells by each pair of modeled/reference states.

Despite the group of measures presented, the main comparison test that can be produced from the data displayed in the contingency matrix is the kappa value, from now on referred to as $k$ Value. This measure indicates the degree of agreement between the modeled map and the reference map. The $k$ Value is based on the difference between the actual agreement in the contingency matrix, i.e. the agreement between modeled and reference situations indicated by the main diagonal, and a chance agreement, indicated by the total of rows and columns.

The closest the $k$ Value of a given contingency matrix is to one the highest the similarity between the modeled and the reference maps is. A kValue of one means an absolute agreement between the two maps. 
Table 2

\section{Contingency matrix and its notation}

Where:

$$
n_{i+}=\sum_{i}^{k} n_{i j}, j=1, \ldots, k
$$

\begin{tabular}{cccccc}
\hline Modeled & \multicolumn{6}{c}{ Reference map column $\mathrm{j}$} & \multirow{2}{*}{ Total row i } \\
\cline { 2 - 5 } map row i & 1 & 2 & $\ldots$ & $\mathrm{k}$ & \\
\hline 1 & $\mathrm{n}_{11}$ & $\mathrm{n}_{12}$ & $\ldots$ & $\mathrm{n}_{1 \mathrm{k}}$ & $\mathrm{n}_{1+}$ \\
\hline 2 & $\mathrm{n}_{21}$ & $\mathrm{n}_{22}$ & $\ldots$ & $\mathrm{n}_{2 \mathrm{k}}$ & $\mathrm{n}_{2+}$ \\
\hline$\ldots$ & $\ldots$ & $\ldots$ & $\ldots$ & $\ldots$ & $\ldots$ \\
\hline $\mathrm{k}$ & $\mathrm{n}_{\mathrm{k} 1}$ & $\mathrm{n}_{\mathrm{k} 2}$ & $\ldots$ & $\mathrm{n}_{\mathrm{kk}}$ & $\mathrm{n}_{\mathrm{k}+}$ \\
\hline $\begin{array}{c}\text { Total } \\
\text { column } \mathrm{j}\end{array}$ & $\mathrm{n}_{+1}$ & $\mathrm{n}_{+2}$ & $\ldots$ & $\mathrm{n}_{+\mathrm{k}}$ & \\
\end{tabular}

$n_{+j}=\sum_{j}^{k} n_{i j}, i=1, \ldots, k$

$\mathrm{n}$ - total number of cells

$\mathrm{k}$ - total number of cell states

OA - Overall accuracy

PA - Producer's accuracy

UA - User's accuracy

$$
\begin{gathered}
O A=\frac{\sum_{i}^{k} n_{i i}}{n}, i=1, \ldots, k \\
P A=\frac{n_{j j}}{n_{+j}}, i=1, \ldots, k \\
U A=\frac{n_{i i}}{n_{i+}}, i=1, \ldots, k
\end{gathered}
$$

The procedure consists in distributing $n$ cells through a $k \times k$ matrix where $k$ is the total number of cell states. Each cell is associated to one of the $k$ cell states within the simulation (usually placed in rows), and to one of the $k$ cell states within the reference data (usually placed in columns). $n_{i j}$ is the number of cells in cell state $i(i=1,2, \ldots, k)$ in simulation and cell state $j(j=$ $1,2, \ldots, k)$ within the set of reference data.

The proportion of cell $i, j$ from the total number of cells $p_{i j}=n_{i j} / n$ is the actual agreement $p_{0}$ of the matrix, is given by

$p_{o}=\sum_{i=1}^{k} p_{i i}$

and the chance agreement is given by

$p_{c}=\sum_{i}^{k} p_{i+} p_{+j}$

where $p_{i+}=\sum_{j=1}^{k} p_{i j}$ and $p_{+j}=\sum_{i=1}^{k} p_{i j}$. Therefore, the $k$ Value is given by

$\hat{K}=\frac{p_{o}-p_{c}}{1-p_{c}}$. 
or

$$
\hat{K}=\frac{n \sum_{i}^{k} n_{i i}-\sum_{i}^{k} n_{i+} n_{+i}}{n^{2}-\sum_{i}^{k} n_{i+} n_{+i}} .
$$

Another important measure that may also be computed from the contingency matrix is the conditional kappa value. Conditional $k$ Value represents the agreement of each cell state inside the matrix. Its value is calculated by the expression

$$
\hat{K}_{i}=\frac{n n_{i i}-n_{i+} n_{+i}}{n n_{i_{+}}-n_{i_{+}} n_{+i}}, i=1,2, \ldots, k .
$$

Because of its strong intuitive character, the use of $k$ Value and its associated statistics is widely used as an assessment of accuracy for image similarity and spatial based model performances. Another way of measuring simulation quality is using form indexes that compare simulated and reference urban forms. An example is the Lee Sallee form index used for example by Clarke et al. (1997). It is a simple relationship between the common area of two different forms and the total area occupied by those forms. Being $A$ an object with a given form and $B$ another object with a form similar to $A$, the Lee Sallee index $L S$ of similarity between $A$ and $B$ is given by the following expression:

$$
L S=\frac{A \cap B}{A \cup B}
$$

Lee Sallee index is equal to one if both objects are equal and is equal to zero if both objects do not have any area in common. The higher the index value is, the better the simulation is.

Finally, it is important to mention the use of fractal dimensions as goodness-of-fit functions for model performance evaluation, commonly applied as measures for CA modeling (Benguigui, Czamanski, Marinov and Portugali, 2000, Herold et al., 2005, Longley and Mesev, 2000, Ménard and Marceau, 2005, White and Engelen, 1993). Complexity associated to CA soon provided the grounds for the use of this type of measures. Cities can be understood as complex systems with complex rules of evolution, integrating the influence of several interdependent factors that are very difficult to model and to control. Several studies published in the 1990s established a series of values for fractal dimensions that where aimed to classify city's morphology (Batty and Longley, 1994, De Keersmaecker, Frankhauser and Thomas, 2003, Frankhauser, 1991, White and Engelen, 1993).

The use of fractal dimensions as measures of performance for CA models depends on the comparison of fractal measures obtained for simulated and for reference maps. White and Engelen (1993) uses sensitivity analyses for different sets of calibration parameters to obtain different urban patterns. Fractal measures are calculated and compared to known values for previously studied cities. The aim is to evaluate the ability of the CA model to generate probable urban patterns. Barredo et al. (2003) uses fractal measures to compare CA-based simulation for Dublin metropolitan area with real values assessed from reference maps of Dublin. Calibration is then achieved when a set of parameters that generates the most similar urban pattern to reality is found. 
Benguigui et al. (Benguigui et al., 2000) presented a method to determine the fractal dimension of an object, based in the correlation between the side of a square in a squared grid, $I$, and the total dimension of the grid that covers the entire object, as depicted in Figure 9. The number corresponding to the surface of the square, $N$, is equal to the relationship between the total area of the grid, $L^{2}$, and the area of the square unit, $l^{2}, N=L^{2} / l^{2}$.

If the object turns out to be fractal, the relationship can be described by the expression $N=(L / l)^{D}$, where $D$ represents the object's fractal dimension. The logarithmic plot of $\ln (N)$ versus $\ln (I)$ yields a straight line with slope $(-D)$ (Figure 10). As an example of the use of this fractal dimension applied to the case of the Tel-A-Viv metropolitan area, Benguigui et al. (2000) conclude that because of the existence of different fractal dimensions for different regions of the study area, that is, different areas presenting different slopes for the relationship described above, it is considered non fractal, therefore heterogeneous.

\section{Figure $9 \quad$ Grid used for a two dimensional object}

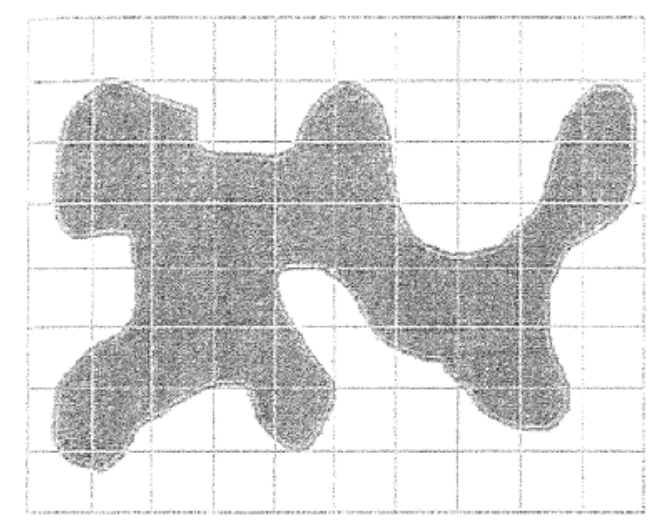

Source: Benguigui et al., 2000
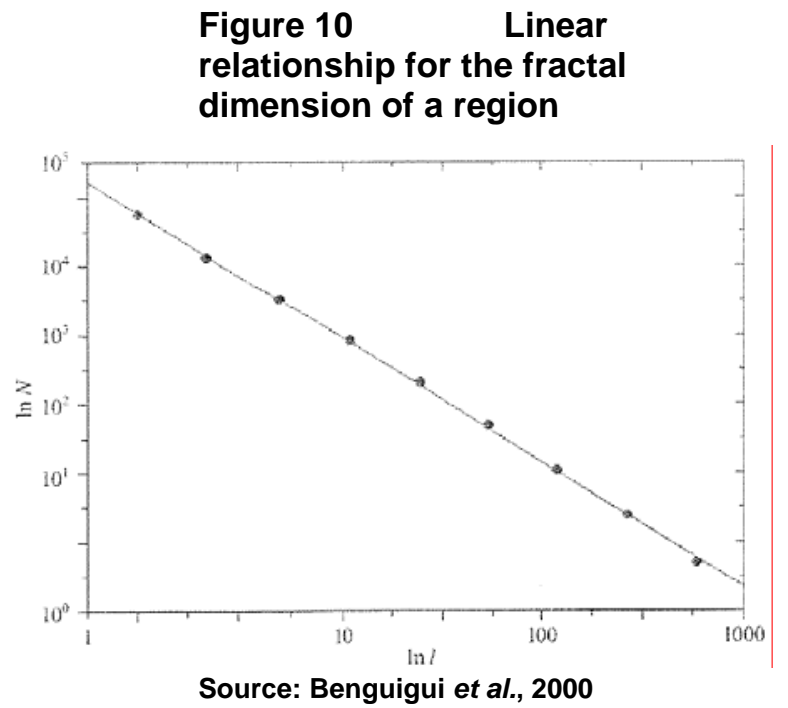

Another method to assess the fractal dimension of an object is presented by White and Engelen (1993) (inspired by the work of Mandelbrot (1983)). This method is based on the evaluation of an object's dimension (expressed, for example, by the number of cells) versus its radius. Cities consist of scattered distributions of urban activities disposed within the territory they occupy. The use of fractal dimensions for cities can be perceived from the parallel established with the Sierpinski carpet problem ${ }^{4}$ depicted in Figure 11. For such objects, it has been shown that

$$
B^{i}=q^{-i D}
$$

where $B$ is the number of cells occupied by the original object (it takes the value of 5 for the Sierpinski problem), $i$ is the step number $(i=1$ in Figure $11 \mathrm{a}, i=2$ in Figure $11 \mathrm{~b}$ and $i=3$ in Figure $11 c$ ), $q$ is the scale reduction factor (it takes the value of $1 / 3$ in Figure 11) and $D$ is the fractal dimension. Solving this Equation 18 for $D$ it can be obtained

\footnotetext{
${ }^{4}$ Cities, of course, present a stochastic behaviour that is totally absent of in the Sierpinski carpet problem.
} 


$$
D=\frac{\log (B)}{\log \left(\frac{1}{q}\right)}
$$

As it can be seen in Figure 11, as the object expands in cell space, the number of cells composing it grows less rapidly than the number of cells in the square area necessary to contain it, so that the object becomes more sparse (White and Engelen, 1993). In particular, the length $L$ of a side of the figure is

$$
L=\left(\frac{1}{q}\right)^{i}
$$

and the total number of cells $B_{T}$ is given by

$$
B_{T}=B^{i}
$$

Figure 11

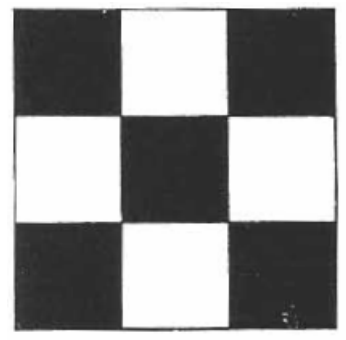

(a)

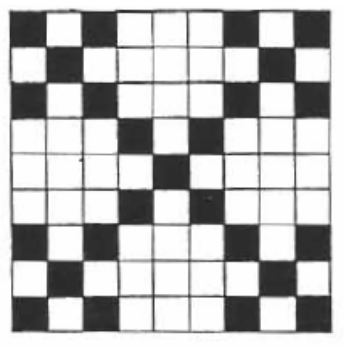

(b)
The Sierpinski carpet

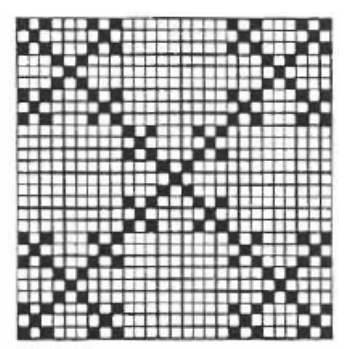

(c)

The relationship between the size of an object (measured in the number of cells) and its diameter (fractal dimension) is given by the expression

$B_{T}=L^{D}$

which can be transformed in a linear relationship by the application of logarithm

$\ln B_{T}=c+D \ln r$

where $c$ is a constant and $r$ is the radius of the object. Equation 23 provides a useful way of estimating the fractal dimension $D$ of a city (considering its stochastic spatial distribution) as it can be obtained from a regression analysis between the variables $B_{T}$ (total number of cells in a given cell state) and $r$ (the radius of its distribution), being its slope the fractal dimension value for that cell state. Note that this measure is location specific, as it depends on the choice of the point of origin for measuring the objects radius. Figure 12 depicts the graphical output of the relationship for the city of Cincinnati for the four land uses considered by White and Engelen (1993). 
The measure of the area-radius relationship yields important information on the urban morphology of a city. The regression equation represented in Figure 12 (b) for industrial land use has a bi-linear behavior, indicating that the city of Cincinnati has a bi-fractal behavior for industry, that is, there are two distinct concentric regions of the city with two different values of the fractal dimension. White and Engelen (1993) also present the values for the radial dimensions of a series of conceptual spatial distributions generated by their CA model and for a set of four US cities, shown in Table 3.

Table 3

Radial dimensions for a set of conceptual cities (cellular group) and US cities

\begin{tabular}{|c|c|c|c|c|c|c|}
\hline \multirow[b]{2}{*}{ Land use } & \multicolumn{3}{|c|}{ Cellular group } & \multicolumn{3}{|l|}{ US city } \\
\hline & 1 & 2 & 3 & Atlanta & Cincinnati & Houston \\
\hline Commerce & 1.09 & 1.17 & 1.03 & 1.00 & 1.10 & 1.24 \\
\hline Industry & 1.85 & 2.41 & 2.72 & 1.97 & 2.11 & 1.51 \\
\hline Housing & 2.29 & 2.96 & 2.77 & 2.12 & 2.51 & 2.76 \\
\hline Other & & & & 2.52 & 3.42 & 2.77 \\
\hline
\end{tabular}

Source: White and Engelen, 1993

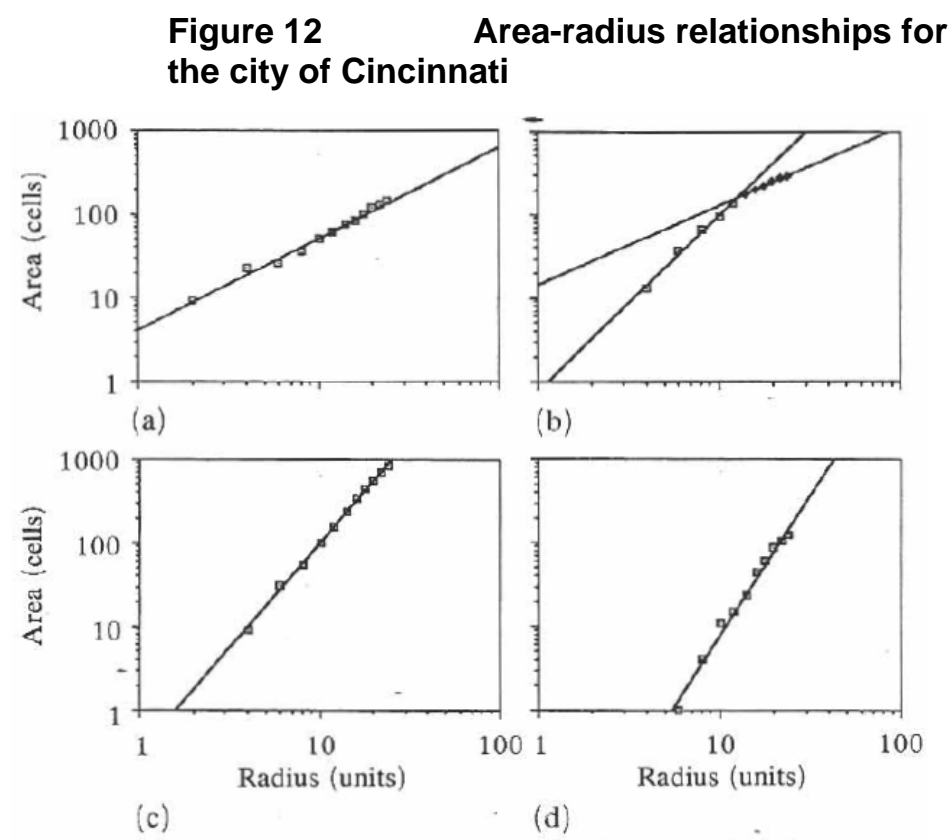

(a) commerce (b) industry (c) housing (d) other land uses

Source: White and Engelen, 1993

This radial dimension measure, as it was referred to by Frankhauser and Sadler (1991), is based on the consideration of a single centre, ignoring the fact that city growth generates a series of new city centers.

Another fractal measure presented by White and Engelen (1993) is the cluster size frequency spectrum. Considering the set of cells occupied by a particular land use in a city as an object formed by a number of clusters of various sizes, it can be found a fractal behavior of the object if there is no characteristic cluster size, this is, if every change of scale maintains the frequency 
ratio of clusters that differ in size by a given factor. This behavior indicates that the object is selfsimilar. The log-log plot of frequency of occurrence of each size against cluster size will be linear if the object is fractal. In Figure 13 is depicted this relationship for commerce for a set of four US cities. Note that Atlanta does not present a linear relationship; hence commerce has no fractal behavior in this case.

Clusters are commonly defined in the literature only by horizontal and vertical adjacencies (White and Engelen, 1993). However, as a city grows, all land uses grow along, making this cluster size frequency measure iteration specific.

These authors also present perimeter-length scaling as a fractal measure used for assessing urban growth. It is based on a property of fractal lines: its measured length increases as the length of the unit with which it is measured decreases. The slope in a log-log relationship between the number of steps required to make one trip around the object's boundary and the length of the step used yields the fractal dimension of the boundary. The close range of values obtained by White and Engelen (1993) for a conceptual city for different moments of the city evolution makes evident that cities are quickly organized into a structure which is maintained as they grow (see Figure 14).

Figure $13 \quad$ Cluster size frequency spectra for commerce in four US cities, in 1960

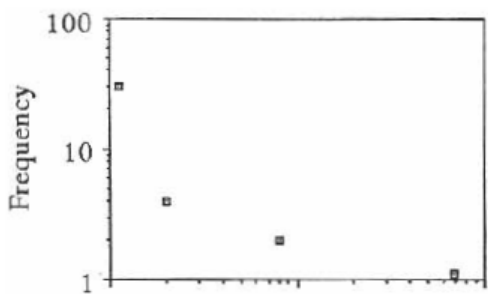

(a)

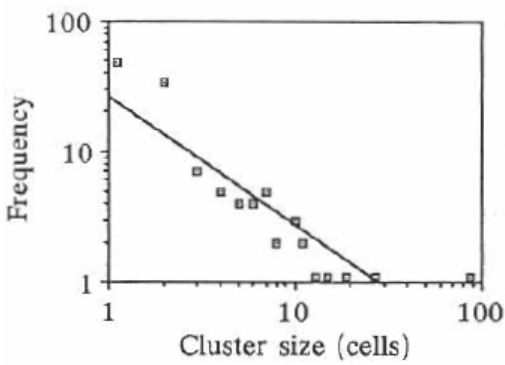

(c)

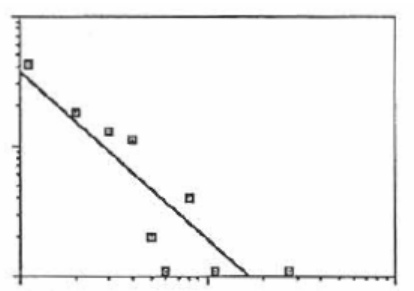

(b)

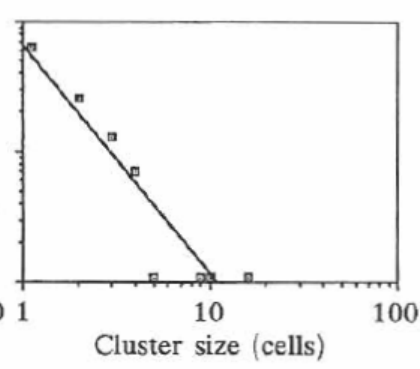

(d)

(a) Atlanta (b) Cincinnati (c) Houston (d) Milwaukee Source: White and Engelen, 1993 
Figure 14

city

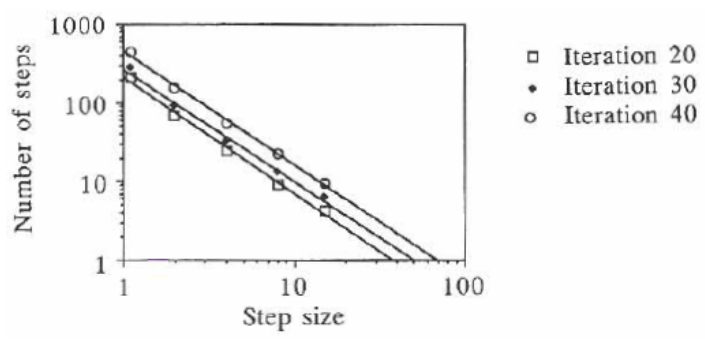

Source: White and Engelen, 1993
Perimeter-length scaling for a conceptual

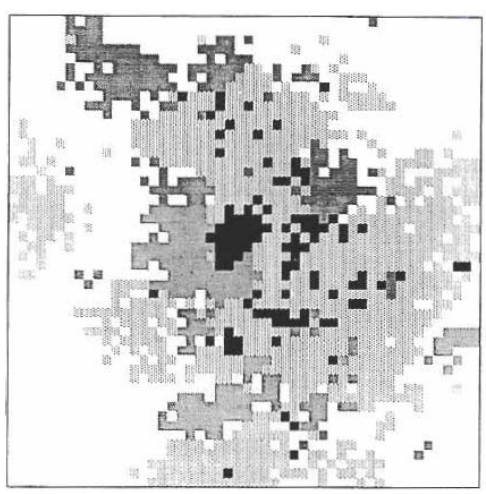

\section{Calibration of CA}

As important as the conceptual development of a model, calibration is aimed to ensure the necessary connection between simulation and reality. No matter how capable a model approach is to simulate real world phenomena - and CA are a very physical method of simulation - it will not produce good simulation without proper calibration.

In the past section a series of measures commonly used to assess CA performance were presented. From visual methods to performance measures and fractal analysis, several approaches are usually mixed to develop calibration procedures. In the present section these approaches will be presented for the major CA models that were developed or are currently under development.

There are two main approaches to calibration: one more dependent on user intervention and another based on optimization techniques. The first one uses visual comparison of simulation and reference maps to determine initial parameter values and sensitivity analysis to improve these values. The second one is based on different techniques aimed to identify the optimal set of parameters without user intervention. The main purpose is to allow the model to establish the correct set of parameters that can simulate complex urban interactions.

SLEUTH calibration (Candau, 2000, Clarke, Hoppen and Gaydos, 1996, Clarke et al., 1997, Silva and Clarke, 2002) is based on a two-step procedure: first, a visual calibration oriented for a broad parameter definition and debugging, based on an user-friendly, real time application that allows the user to stop the simulation and to modify a given parameter, analyzing model's sensitiveness to each parameter; second, a brute force calibration procedure where multiple runs are produced in order to generate enough model data to statistically compare reference data. Visual calibration is considered useful to establish meaningful ranges of values for parameters. A set of statistical measures are displayed in real time to allow the user to understand how the model reacts to a given parameter change. The second stage is much more complex and is based on a sequential procedure of consecutive runs of the model aiming to obtain a good set of parameters at three levels of calibration (coarse, fine and final). 
Considering the results obtained from a set of thirteen metrics, each phase is fed with the best scoring results for the set of control parameters, weighted with a set of user-defined control parameters (for a detailed descriptions see (Clarke et al., 1996)).

The Li and Yeh CA-based model (Li and Yeh, 2001) used artificial neural networks (ANN) not only to simulate urban change but also for calibration purposes. The use of ANN for model calibration is presented as a solid alternative to other methods that are user-dependent and computationally expensive. The model uses the concept of transition probability that is multiplied by a stochastic factor aimed to deal with uncertainty. As it can be seen in Figure 7, the ANN model uses empirical data obtained from GIS and remote sensing to train the network in order to establish values for development probabilities for each land use. It relates multiple cell attributes (obtained from GIS) and growth history (obtained from remote sensing) to calculate a set of calibration parameters that are subsequently used by CA-based model to simulate urban change. Calibration is produced with selected sample data sets, using a stratified sampling method to assign a certain number of observations for each category to be evaluated. Results showed that the method succeeded in achieving logical results for site attributes. However, training process must be controlled in its duration in order to stop the process when the network begins to over-train.

The model designed by Barredo et al. (2003) was calibrated through a simple process of sensitivity analysis of the calibration factors considered. After a visual analysis of simulation results, calibration factors were carefully altered and new simulations were produced and their results assessed. Fractal measures were calculated and compared with observed values for the case study. This process can be considered excessively simple because it does not consider the combined effects of the entire set of parameters. Urban phenomena are a result of several interactive behaviors and the analysis of each of these behaviors by itself considering the others as fixed may produce significant distortions and reduce the representativeness of the model.

The model proposed by Pinto (2006) was calibrated by using an optimization procedure based on the Particle Swarm (PS) algorithm (Eberhart and Kennedy, 1995, Parsopoulos and Vrahatis, 2002). The choice for an optimization approach is related to the strong degree of interdependence that urban phenomena have. Optimization is aimed to find a near-optimum solution for a set of model parameters; these calibration parameters are interdependent, therefore they should not be considered isolated, as in sensitivity analyses, because of the risk of missing important characteristics of model behavior. PS is a very intuitive algorithm that uses a set of particles - each particle is a set of calibration parameters - to "fly" over a space of solutions, with the group of particles concurring for an optimum "flight layout" - which correspond to the near-optimum set of calibration parameters. 


\section{Conclusion}

The main goal of this paper was to present an introductory literature survey on cellular automata (CA) and urban studies through the presentation and discussion of issues regarding model formulations, evolutions, and applications. Rather than drawing any conclusions, it is pertinent to comment the use of CA and its future as an urban modeling tool.

It is clear that the use of CA is, on the one hand, a promising approach for developing good modeling tools that could simulate urban phenomena to assist planning processes. On the other hand, there is a long way to go before these models can simulate correctly human behaviors and urban complexity. Further improvements must be made towards models that integrate urban policy testing and complexity parameters. In particular, important components of CA, such as cell space or neighborhood, must also undergo further research.

Nevertheless, CA have a promising future as an urban simulation tool. In fact, microsimulation is now presented as the new paradigm of modeling for a wide set of phenomena. But a new question regarding CA must be posed: will future models still be classified as standard geographic CA or will we have cellular-based models, as a result of years and years of consecutive relaxations from the classical CA formulation? The next generation of CA models, probably linked to higher resolution simulation and based on more powerful computational tools will provide an answer to that. 


\section{BIBLIOGRAFÍA}

Barredo, J., M. Kasanko, N. McCormick and C. Lavalle. 2003. Modelling dynamic spatial processes: simulation of urban future scenarios through cellular automata, Landscape and Urban Planning, 64 145-160.

Batty, M. 1994. A chronicle of scientific planning - The Anglo-American modelling experience, Journal of the American Planning Association, 60 (1) 7-16.

2005. Cities and complexity : understanding cities with cellular automata, agent-based models, and fractals, MIT, Cambridge, Mass. ; London. xxiii, 565.

Batty, M., H. Couclelis and M. Eichen. 1997. Editorial: Urban systems as cellular automata, Environment and Planning B: Planning and Design, 24 159-164.

Batty, M. and P. Longley. 1994. Fractal cities: a geometry of form and function, Academic Press, London ; San Diego. xxi, 394.

Batty, M. and Y. Xie. 1997. Possible urban automata, Environment and Planning B: Planning and Design, 24 175-192.

Batty, M., Y. Xie and Z. Sun. 1999. Modelling urban dynamics through GIS-based cellular automata, Computers, Environment and Urban Systems, 23 205-233.

Benenson, I. and P.M. Torrens. 2004. Geosimulation - Automata-based modeling of urban phenomena, John Wiley \& Sons Ltd, Chechester. xxiii, 287.

Benguigui, L., D. Czamanski, M. Marinov and Y. Portugali. 2000. When and where is a city fractal?, Environment and Planning B: Planning and Design, 27 507-519.

Candau, J. 2000. Calibrating a cellular automaton model of urban growth in a timely manner. Proceedings of 4th International Conference on Integrating GIS and Environmental Modeling (GIS/EM4). Ed. Banff, Alberta, Canada, September 2-8, 2000.

Clarke, K. 2002. Land Use Change Modeling Using SLEUTH. Proceedings of Advanced Training Workshop on Land Use and Land Cover Change Study. Ed. Taiwan, National Central University/National Taiwan University/ START, December 9-20th. 525-573.

Clarke, K., S. Hoppen and L. Gaydos. 1996. Methods and Techniques for Rigourous Calibration of a Cellular Automaton Model of Urban Growth. Proceedings of Third International Conference/Workshop on Integrating Geographic Information Systems and Environmental Modeling. Ed. Santa Fe, NM, January 21-25th.

. 1997. A self-modifying cellular automaton model of historical urbanization in the San Francisco Bay area, Environment and Planning B: Planning and Design, 24 247-261.

Couclelis, H. 1985. Cellular worlds: a framework for modelling micro-macro dynamics, Environment and Planning A, 17 585-596.

1997. From cellular automata to urban models: new principles for model development and implementation, Environment and Planning B: Planning and Design, 24 165-174. 
Couto, P. 2003. Assessing the accuracy of spatial simulation models, Ecological Modelling, 167 181-198.

De Keersmaecker, M.-L., P. Frankhauser and I. Thomas. 2003. Using fractal dimensions for characterizing intra-urban diversity: the example of Brussels, Geographical Analysis, 35 (4) 310 328.

Eberhart, R. and J. Kennedy. 1995. A New Optimizer Using Particle Swarm Theory. Proceedings of Sixth International Symposium on Micro Machine and Human Science. Ed. 3943

Eloranta, K. 1997. Critical growth phenomena in cellular automata, Physica D: Nonlinear Phenomena Lattice Dynamics, 103 (1-4) 478-484.

Frankhauser, P. 1991. Aspects fractals des structures urbaines, L'Espace Géographique, $145-$ 69.

Frankhauser, P. and R. Sadler. 1991. Fractal analysis of agglomerations. Proceedings of Second International Colloquium of the Sonderforshungsbereich 230: Natural Structures Sonderforshungsbereich 230. Ed. Stuttgard, 57-65

Gerling, R.W. 1990. Classification of triangular and honeycomb cellular automata, Physica A: Statistical and Theoretical Physics, 162 (2) 196-209.

Herold, M., H. Couclelis and K.C. Clarke. 2005. The role of spatial metrics in the analysis and modelling of urban land use change, Computers, Environment and Urban Systems, 29 369-399. Klosterman, R. 1994. Large-Scale urban models - Retrospect and prospect, Journal of the American Planning Association, 60 (1) 3-6.

Lee, D. 1973. Requiem for large-scale models, Journal of the American Planning Association, 39 (3) 163-178.

Levy, S. 1992. Artificial life : the quest for a new creation, Pantheon Books, New York. viii, 390.

$\mathrm{Li}, \mathrm{X}$. and A. Yeh. 2001. Calibration of cellular automata by using neural networks for the simulation of complex urban systems, Environment and Planning A, 33 1445-1462.

Lindenmayer, A. 1968. Mathematical models for cellular interaction in development, Parts I and II, Journal of Theoretical Biology, 18 280-315.

Longley, P.A. and V. Mesev. 2000. On the measurement and generalisation of urban form, Environment and Planning A, 32 473-488.

Mandelbrot, B. 1983. The Fractal Geometry of Nature, W. H. Freeman, New York. 480.

Ménard, A. and D.J. Marceau. 2005. Exploration of spatial scale sensitivity in geographical cellular automata, Environment and Planning B: Planning and Design, 32 693-714.

O'Sullivan, D. 2001a. Exploring Spatial Process Dynamics Using Irregular Cellular Automaton Models, Geographical Analysis, 33 (1) 1-18.

2001b. Graph-based cellular automaton models of urban spatial processes. PhD Thesis, Bartlett School of Architecture and Planning, University College, London. 317

Parsopoulos, K.E. and M.N. Vrahatis. 2002. Recent approaches to global optimization problems through Particle Swarm Optimization, Natural Computing, 1 235-306. 
Pinto, N. 2006. A microsimulation approach for modelling the growth of small urban areas. MSc Thesis, Civil Engineering Department, School of Engineering of the University of Porto, Porto. 156

Putman, S.H. and C. Shih-Liang. 2001. The METROPILUS planning support system: urban models and GIS, Planning Support Systems: Integrating Geographical Information Systems, Models and Visualization Tools. Ed. Brail, RK and RE Klosterman. ESRI Press, Redlands, CA. 99-128.

Semboloni, F. 1997. An urban and regional model based on cellular automata, Environment and Planning B-Planning \& Design, 24 (4) 589-612.

2000. The growth of an urban cluster into a dynamic self-modifying spatial pattern, Environment and Planning B-Planning \& Design, 27 (4) 549-564.

Silva, E. and K.C. Clarke. 2002. Calibration of the SLEUTH urban growth model for Lisbon and Porto, Portugal, Computers, Environment and Urban Systems, 26 525-552.

Takeyama, M. and H. Couclelis. 1997. Map dynamics: integrating cellular automata and GIS through Geo-Algebra, International Journal of Geographical Information Science, 11 (1) 73-91.

Tobler, W. 1970. A Computer Movie Simulating Urban Growth in the Detroit Region, Economic Geography, 46 (2) 234-240.

1979. Cellular geography, Philosophy in Geography. Ed. Gale, S and G Olsson. D. Reidel, Boston. 379-386.

Torrens, P. 2000. How cellular models of urban systems work (1. Theory). CASA Working Papers Series. Accessed in November 2005

Torrens, P. and D. O'Sullivan. 2001. Editorial: Cellular automata and urban simulation: where do we go from here?, Environment and Planning B: Planning and Design, 28 163-168.

Vandergue, D., J.-P. Treuil and D. Drogoul. 2000. Modelling urban phenomena with cellular automata. Proceedings of Applications of Simulation to Social Science. Ed. Ballot, G and G Weisbuch. Paris, 127-140

Wagner, D.F. 1997. Cellular automata and geographic information systems, Environment and Planning B: Planning and Design, 24 219-234.

Ward, D., A. Murray and S. Phinn. 2003. Integrating spatial optimization and cellular automata for evaluating urban change, The Annals of Regional Science, 37 131-148.

White, R. and G. Engelen. 1993. Cellular Automata and fractal urban form: a cellular modelling approach to the evolution of urban land-use patterns, Environment and Planning A, 25 11751199.

1997. Cellular automata as the basis of integrated dynamic regional modelling, Environment and Planning B: Planning and Design, 24 235-246.

2000. High-resolution integrated modelling of the spatial dynamics of urban and regional systems, Computers, Environment and Urban Systems, 24 383-400.

Wolfram, S. 1983. Statistical mechanics of cellular automata, Reviews of Modern Physics, $\mathbf{5 5}$ (3) $601-644$.

1984. Computation theory of cellular automata, Communications in Mathematical

Physics, 96 (1) 15-57. 
2005. Historical notes: history of cellular automata. Wolfram Science. Accessed in 2005Xie, Y. 1996. A generalized model for cellular urban dynamics, Geographical Analysis, $284350-$ 373.

Zeigler, B.P. 1976. Theory of Modeling and Simulation, Wiley, New York. 